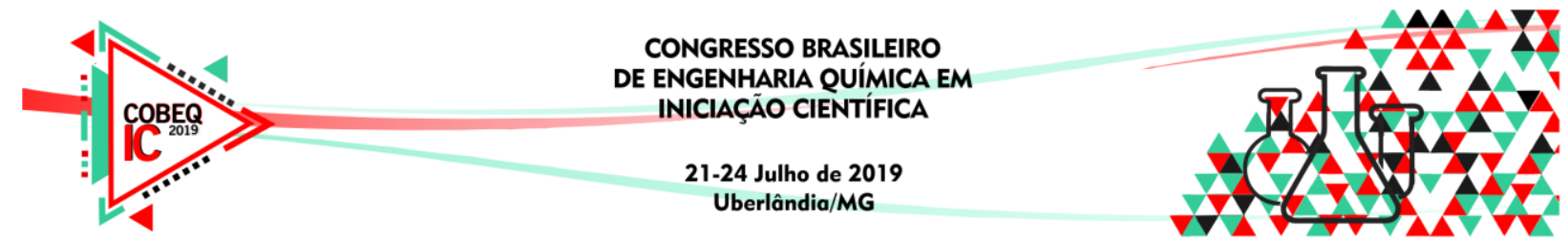

\title{
INFLUENCIA DA MISTURA DE PARTÍCULAS DE DIFERENTES DENSIDADES SOBRE A FLUIDODINÂMICA NO INTERIOR DE UM LEITO DE JORRO
}

\author{
H. B. BEZERRA ${ }^{1}$, J. N. S. MORAIS ${ }^{1}$ e D. A. SANTOS $^{1}$ \\ ${ }^{1}$ Universidade Federal de Goiás, Instituto de Química, Curso de Engenharia Química \\ E-mail para contato: hanielbraga@ hotmail.com
}

\begin{abstract}
RESUMO - A aplicação da técnica de leito de jorro proporciona um satisfatório contato fluido-partícula, sendo essa característica de fundamental importância nos processos de transferência de calor e massa. Com o objetivo de compreender melhor os fenômenos pertinentes à operação de um leito de jorro, este trabalho teve por objetivo analisar a influência da mistura de partículas com diferentes densidades sobre a condição de mínimo jorro, comparando, desta forma, as curvas características da mistura em questão com as obtidas com as monopartículas que a compõem, em diferentes alturas de leito estático. O comportamento da curva característica da mistura foi semelhante àquele da esfera de maior densidade, visto que ocorreu segregação destas para a região de jorro, dominando, desta forma, a dinâmica do movimento em seu interior e, consequentemente, a velocidade de mínimo jorro. Já as de menor densidade migraram para a região anular, o que levou a uma menor influência destas sobre a condição de mínimo jorro. Adicionalmente, como era de se esperar, o aumento da altura de leito estático causou um aumento nos valores de velocidade de mínimo jorro da mistura.
\end{abstract}

\section{INTRODUÇÃO}

O leito de jorro foi concebido, inicialmente, como uma versão modificada do leito fluidizado com o intuito de melhorar o contato fluido-partícula em seu interior, se aproveitando da geometria cônica, que faz com que todas as partículas participem da mistura dentro do equipamento. Dessa forma, são evitadas possíveis "zonas mortas", sendo essas, quaisquer regiões dentro do equipamento em que as partículas não participam ativamente da interação com o fluido (Mathur e Epstein, 1974).

De acordo com Garim (1998), para se estudar a fluidodinâmica de um leito de jorro, é necessária a obtenção da sua curva característica. Tal curva representa a transição entre um leito estático e um regime de jorro estabelecido e é melhor descrita quando se plota a queda de pressão no leito em função da velocidade do fluido (Mathur e Epstein 1974). A Figura 1 a seguir exemplifica o comportamento da curva característica de um leito de jorro (queda de pressão no leito versus velocidade do fluido na entrada do leito). 


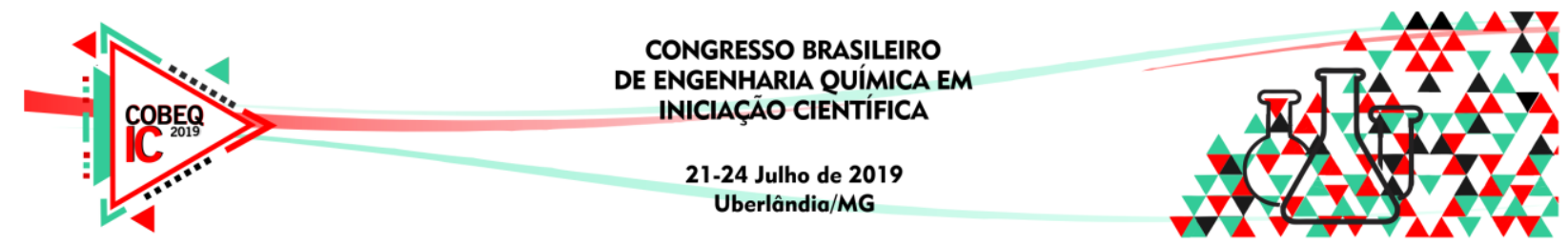

Figura 1 - Comportamento da curva característica de um leito de jorro: queda de pressão no leito versus velocidade do fluido na entrada do leito (Bacelos, 2006, apud Santos, 2011).

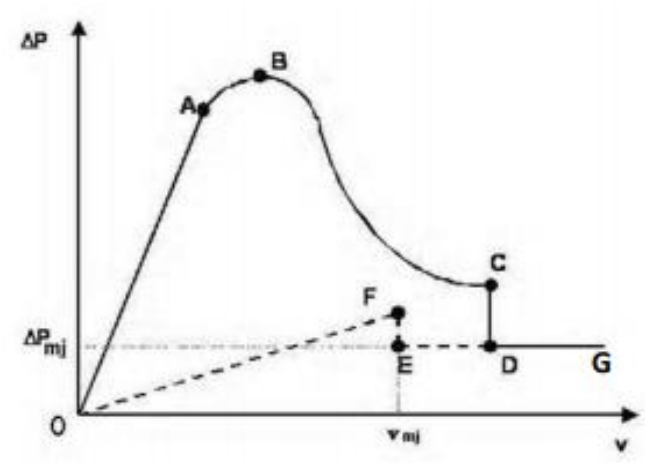

Dadas as condições de operação do leito de jorro, é esperado que as curvas características se alterem, a depender da altura do leito estático e das características físicas das partículas que o compõem. Com base na Figura 1, o aumento da velocidade do fluido na entrada do leito causa um aumento na queda de pressão, cujo comportamento inicial é linear, o que caracteriza um leito estático de partículas (segmento OA).

Aumentando-se a velocidade do fluido, observa-se que a queda de pressão alcança um valor máximo e diminui com o aumento da velocidade do fluido até atingir um valor de queda de pressão aproximadamente constante. Esta região de pressão aproximadamente constante caracteriza, aproximadamente, a velocidade de mínimo jorro, a qual pode ser estabelecida, mais precisamente, por visualização da iminente formação da fonte de partículas. A curva obtida pelo aumento da velocidade é conhecida como "curva de ida". De forma contrária, a curva obtida pela diminuição da velocidade é denominada de "curva de volta". O leito de jorro é conhecido por apresentar o fenômeno de histerese, ou seja, as curvas de ida e volta se diferem.

Também é esperado que a velocidade de mínimo jorro aumente com o aumento da altura de leito estático e com o uso de partículas mais densas, para uma mesma altura de leito estático, visto que a perda de carga será maior nestes casos. Porém, o efeito do aumento ou da diminuição da velocidade de mínimo jorro para mistura de partículas de diferentes propriedades físicas depende do fenômeno da segregação, que deve ser cuidadosamente investigado.

Logo, com base no exposto, este trabalho teve como objetivo principal a investigação da mistura de partículas de diferentes densidades sobre a fluidodinâmica no interior de um leito de jorro. Para tal, as curvas características da mistura em questão foram comparadas com as obtidas com as monopartículas que a compõem, em diferentes alturas de leito estático.

\section{MATERIAIS E MÉTODOS}

Para a obtenção dos dados experimentais, foi utilizada uma unidade de leito de jorro presente no "Laboratório de Sistemas Particulados e Fluidodinâmica Computacional (SisParTec)" do Instituto de Química, Departamento de Engenharia Química, da Universidade Federal de Goiás (Figura 2). 


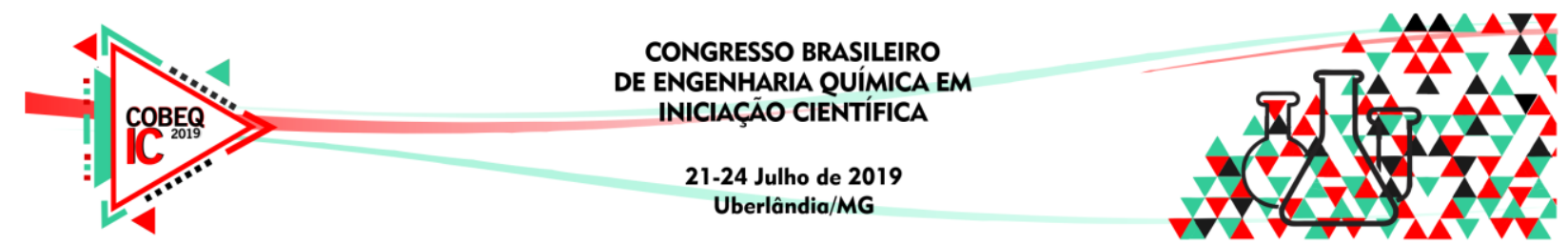

Figura 2 - Unidade experimental de leito de jorro e seus periféricos.

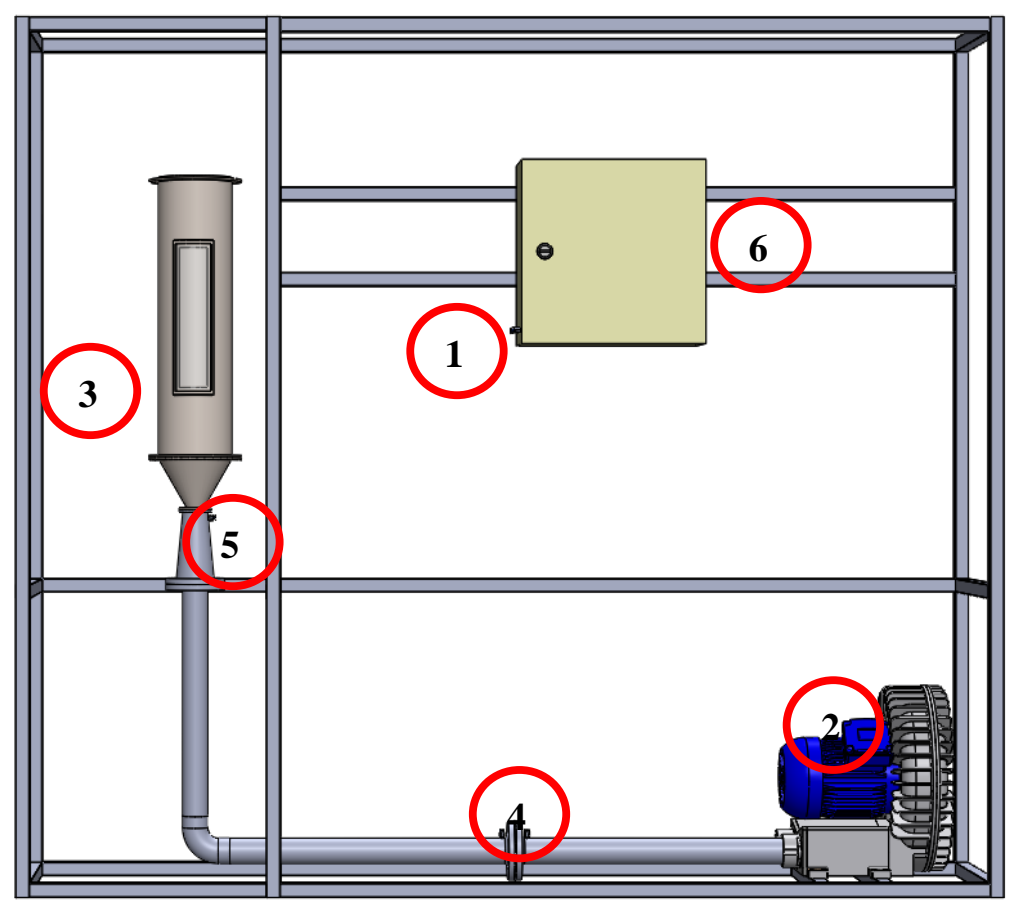

A unidade do leito de jorro mostrada na Figura 2 é composta por: 1. Entrada de aquisição de pressões por mangueiras pneumáticas com engate rápido; 2. Motor soprador radial de 4,0cv de potência, trifásico de baixa rotação; 3 . Estrutura do leito de jorro; 4 . Ponto de aquisição de pressão a montante e a jusante da placa de orifício para a medida da velocidade de escoamento do fluido; 5 . Aquisição de pressão na entrada do leito de jorro com engate rápido rosqueado por conector pneumático; 6 . Painel para controle do motor contendo inversor de frequências CFW500 de 5cv, placa de aquisição de dados Arduino UNO e sensores de pressão e temperatura.

Como material particulado, foram utilizadas esferas de alta precisão de poliacetal e de vidro. As características físicas das partículas, assim como a geometria do leito de jorro, são dispostas na Tabela 1 a seguir:

Tabela 1 - Característica física dos materiais particulados e da geometria do leito de jorro utilizadas durante os experimentos.

\begin{tabular}{c|c|c}
\hline \multirow{4}{*}{ Partículas } & Partículas utilizadas & Vidro e Poliacetal \\
& Mistura (v/v) & $50 \%$ \\
& Diâmetro das partículas & $0,004 \mathrm{~m}$ \\
& Massa específica Vidro & $2500 \mathrm{~kg} / \mathrm{m}^{3}$ \\
& Massa específica Poliacetal & $1400 \mathrm{~kg} / \mathrm{m}^{3}$ \\
\hline \multirow{3}{*}{ Leito de } & Diâmetro da parte cilíndrica & $0,1476 \mathrm{~m}$ \\
jorro & Diâmetro da entrada do leito & $0,020 \mathrm{~m}$ \\
& Altura da parte cônica & $0,110 \mathrm{~m}$ \\
& Altura da parte cilíndrica & $0,400 \mathrm{~m}$ \\
\hline
\end{tabular}




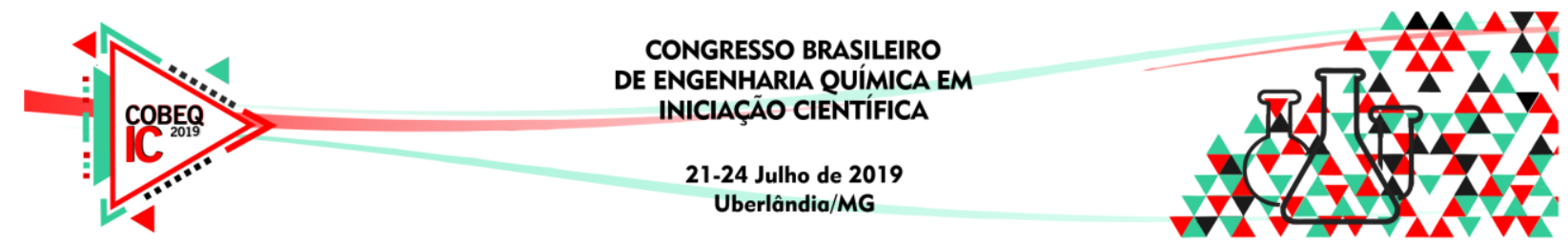

$\mathrm{O}$ ar $\left(\mu=1,81 \times 10^{-5}\right.$ Pa.s e $\left.\rho=1,204 \mathrm{~kg} / \mathrm{m}^{3}\right)$ foi utilizado como fluido de arraste. Todas as curvas características foram obtidas quando da diminuição da velocidade do ar na entrada do leito ("curva de volta").

\section{RESULTADOS E DISCUSSÃO}

Com o intuito de se investigar o efeito da mistura de partículas de diferentes densidades sobre a fluidodinâmica no interior de um leito de jorro, a curva característica da mistura de partículas de poliacetal e vidro (50\% em volume) foi comparada com as respectivas curvas das monoparticulas, como pode ser visto na Figura 3 a seguir.

FIGURA 3 - Curvas características para as monopartículas de vidro e poliacetal e para a mistura de partículas (50\% em volume), para altura de leito estático de $0,110 \mathrm{~cm}$

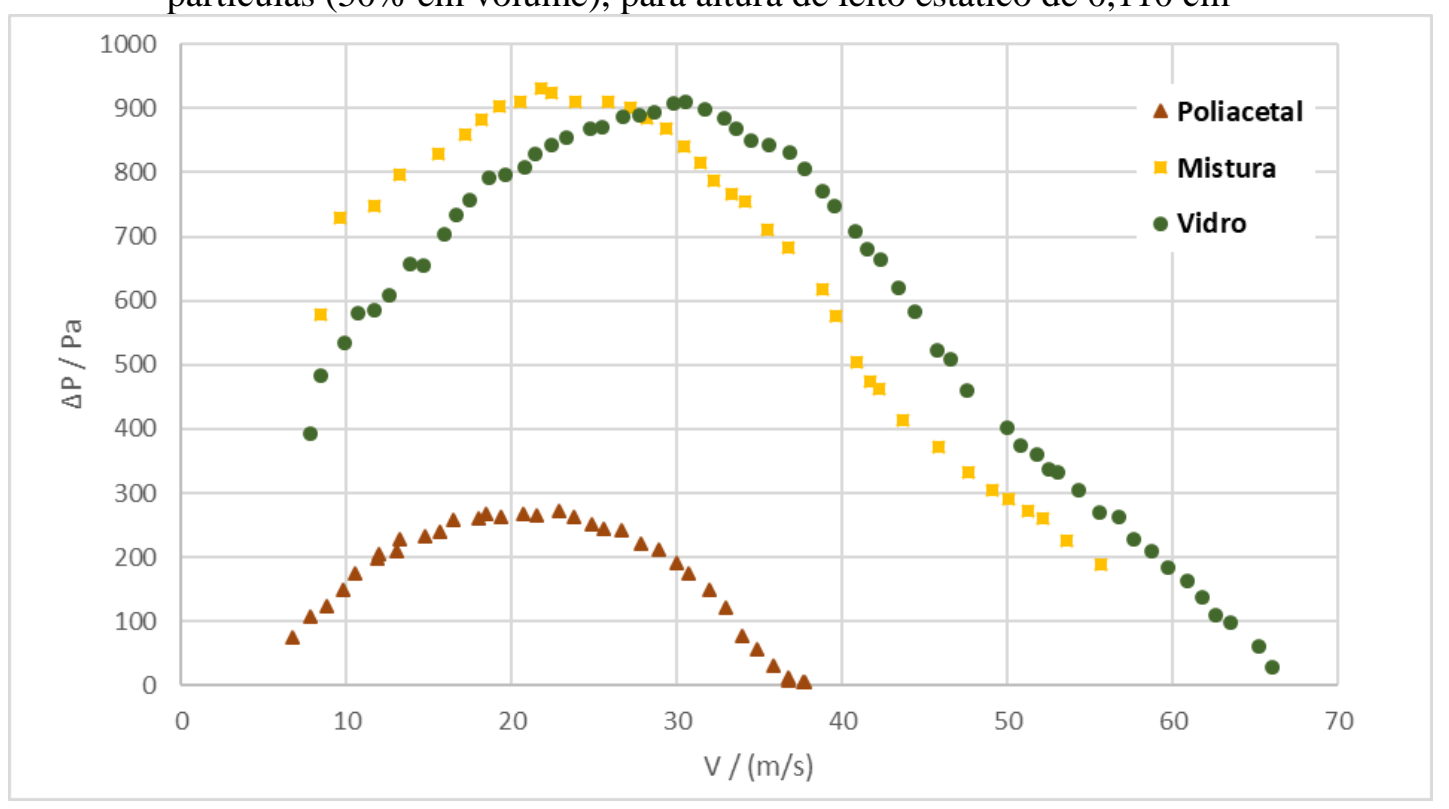

Independente da configuração de partículas, o comportamento de todas as curvas características se mostrou coerente, fisicamente, com o apresentado na Figura 1. No que diz respeito às curvas das monopartículas, como esperado, os maiores valores de máxima queda de pressão no leito e de velocidade de mínimo jorro, foram observados para as esferas de vidro, visto que possuem uma maior densidade e, consequentemente, o leito estático possui uma maior perda de carga (Figura 3).

Já quando da mistura das partículas, o comportamento da curva característica da mistura foi semelhante àquele da esfera de maior densidade (esfera de vidro) (Figura 3). Isto pode ser explicado devido ao fato de que, no início do experimento ocorreu a segregação das partículas mais densas para a região de jorro, dominando, desta forma, a dinâmica do movimento no interior do leito de jorro e, consequentemente, a caracterização da velocidade de mínimo jorro e máxima queda de pressão. Já as de menor densidade (poliacetal) migraram para a região anular, o que levou a uma menor influência destas sobre a condição de mínimo jorro e máxima queda de pressão. 

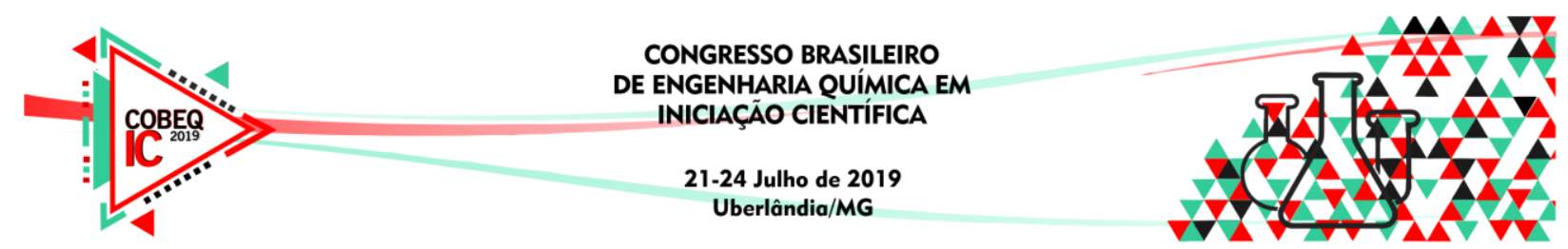

Foram obtidas, também, como pode ser visto na Figura 4, curvas características para a mistura (50\% em volume) sob diferentes condições de leito estático $(9,8 \mathrm{~cm} ; 11,0 \mathrm{~cm}$; e 13,4 $\mathrm{cm}$ de altura de leito estático).

FIGURA 4 - Curvas características para a mistura de partículas de poliacetal e vidro $(50 \% \mathrm{em}$ volume), para diferentes alturas de leito estático: $9,8 \mathrm{~cm}, 11,0 \mathrm{~cm}$ e 13,4 cm.

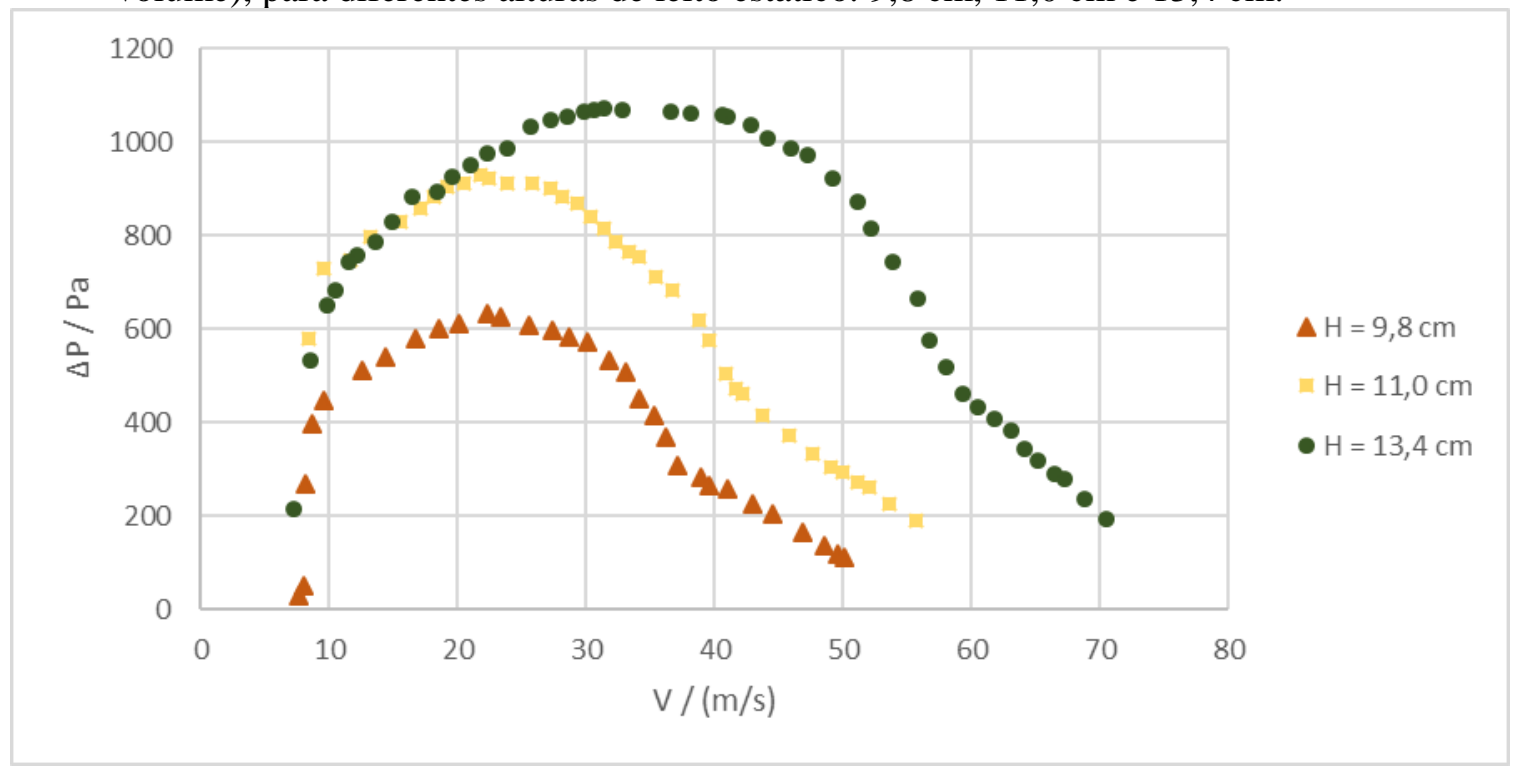

Os correspondentes valores de velocidade de mínimo jorro para todas as condições mostradas nas Figuras 3 e 4 são também dispostos, para uma melhor comparação, na Tabela 2 a seguir.

Tabela 2 - Dados de velocidade de mínimo jorro para as monopartículas e para a mistura (50\% em volume) sob diferentes alturas de leito estático.

\begin{tabular}{c|c|c}
\hline Composição do leito & Altura de leito estático $(\mathrm{m})$ & Velocidade de mínimo jorro (m/s) \\
\hline Poliacetal & 0,110 & 33,87 \\
\hline Vidro & 0,110 & 45,54 \\
\hline \multirow{2}{*}{ Mistura } & 0,098 & 37,49 \\
& 0,110 & 41,18 \\
& 0,134 & 58,32 \\
\hline
\end{tabular}

Como pode ser observado na Figura 4 e na Tabela 2, o aumento da altura de leito estático causou um aumento nos valores de velocidade de mínimo jorro e de máxima queda de pressão, como era de se esperar, em virtude da maior perda de carga. 


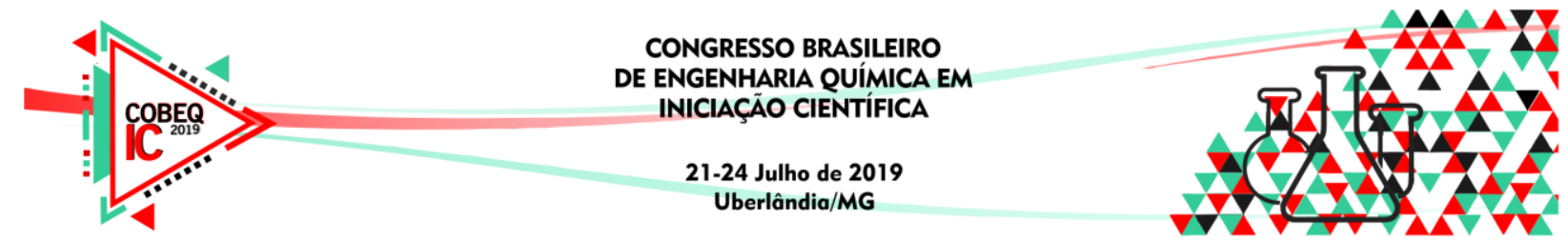

\section{CONCLUSÃO}

Foi possível analisar a influência da mistura de partículas com diferentes densidades sobre a condição de mínimo jorro e de máxima queda de pressão, comparando, desta forma, as curvas características da mistura em questão com as obtidas com as monopartículas que a compõem, em diferentes alturas de leito estático. O comportamento da curva característica da mistura foi semelhante àquele da esfera de maior densidade, visto que ocorreu segregação destas para a região de jorro, dominando, desta forma, a dinâmica do movimento em seu interior e, consequentemente, a velocidade de mínimo jorro. Já as de menor densidade migraram para a região anular, o que levou a uma menor influência destas sobre a condição de mínimo jorro. Adicionalmente, como era de se esperar, o aumento da altura de leito estático causou um aumento nos valores de velocidade de mínimo jorro da mistura.

Logo, o efeito do aumento ou da diminuição dos valores de velocidade de mínimo jorro e máxima queda de pressão para mistura de partículas de diferentes propriedades físicas depende do fenômeno da segregação, que deve ser cuidadosamente investigado.

\section{REFERÊNCIAS}

BACELOS, M. S. Análise do comportamento da fluidodinâmica do leito de jorro com misturas de tamanho de partículas. Tese de Doutorado, PPGEQ/UFSCAR - São Carlos/SP, 2006, 113p.

GARIM, M.M.; FREIRE, J.T. Uma análise das curvas características de leitos fluidizados e vibrofluidizados utilizando as curvas de desvio-padrão das medidas realizadas. In: congresso brasileiro de sistemas particulados - enemp, 14., 1998, Teresópolis. Anais... Rio de Janeiro: DTQ/UFRRJ, 1998. v.1, p.253-9.

MATHUR, K. B.; EPSTEIN, N., Spouted beds. Academic Press Inc. LTD., New York, 304p., 1974.

SANTOS, D. A. Contribuições ao estudo da fluidodinâmica em leito de jorro - estudos experimentais e de simulação via CFD. Uberlândia: UFU - Faculdade de Engenharia Química, Universidade Federal de Uberlândia, 2011. Dissertação (Mestrado). 